\title{
Bacterial Infections in Cirrhotic Patients in a Tertiary Care Hospital
}

\author{
Vivek A. Lingiah*(1) and Nikolaos T. Pyrsopoulos*(1) \\ Department of Medicine, Division of Gastroenterology and Hepatology, Rutgers University, \\ New Jersey Medical School, Newark, NJ, USA
}

\begin{abstract}
Background and Aims: Patients with cirrhosis are immunocompromised and at higher risk of developing infections compared to the general population. The aim of this study was to assess the incidence of infections in cirrhotic patients in a large academic liver center and investigate potential associations between infections, bacteria isolated, therapeutic regimens used, and mortality. Methods: This was a retrospective chart review study, including 192 patients. All patients had a diagnosis of cirrhosis and were admitted to University Hospital. Information collected included demographics, etiology of cirrhosis, identification of bacteria from cultures, multidrug-resistant (MDR) status, antibiotics administered, intensive care unit (ICU) admission, and patient mortality. Results: Infections were present in 105 (54.6\%) patients, and $60(31.2 \%)$ patients had multiple infections during a hospitalization(s) for infections. A total of 201 infections were identified. Urinary tract infections (UTIs) were the most common infection $(37.8 \%)$, followed by bacteremia $(20.4 \%)$, pneumonia $(12.9 \%)$, spontaneous bacterial peritonitis (SBP) $(11.9 \%)$, abscess/cellulitis $(6.0 \%)$, infectious diarrhea $(6.0 \%)$, and other (5.0\%). Escherichia coli was the most common bacteria isolated (13.4\%), both among sensitive and MDR infections. MDR bacteria were the cause for $41.3 \%$ of all infections isolated. Fungi accounted for $9.5 \%$ of infections. $21.9 \%$ of patients had decompensation from their infection(s) that required ICU care, and $14.6 \%$ of patients died during hospitalization or soon after discharge. Conclusions: The incidence of infections in cirrhotic patients is much higher than in their non-cirrhotic counterparts $(54.6 \%)$, even higher than prior studies suggest. As many of these infections are caused by MDR bacteria and fungal organisms, stronger empiric antibiotics and antifungals should be considered when initially treating this
\end{abstract}

Keywords: Cirrhosis; Bacterial infections; Multidrug resistance; Fungal infections.

Abbreviations: AIDS, acquired immune deficiency syndrome; $\mathrm{CI}$, confidence interval; CRE, carbapenem-resistant Enterobacteriaceae; EASL, European Association for the Study of the Liver; ESBL, extended spectrum beta lactamase; GNB, Gram-negative bacilli; HIV, human immunodeficiency virus; ICU, intensive care unit; Ig, immunoglobulin; MDR, multidrug-resistant; MELD, model for endcare unit; Ig, immunoglobulin; MDR, multidrug-resistant; MELD, model for endstage liver disease; MRSA, methicillin-resistant Staphylococcus aureus; NAC-
SELD, North American Consortium for the Study of End-Stage Liver Disease; $\mathrm{OR}$, odds ratio; RES, reticuloendothelial system; RR, relative risk; SBP, spontaneous bacterial peritonitis; SIBO, small intestinal bacterial overgrowth; SIRS systemic inflammatory response syndrome; SOFA, sequential organ failure as sessment; VRE, vancomycin-resistant enterococci; UTI, urinary tract infection. Received: 16 August 2020; Revised: 15 November 2020; Accepted: 05 DeReceived: 16

* Correspondence to: Nikolaos T. Pyrsopoulos and Vivek A. Lingiah, Division of Gastroenterology and Hepatology, Rutgers University, New Jersey Medical School, MSB H-355, 185 S Orange Ave, Newark, NJ 07103, USA. Tel: +1-973972-5252, Fax: +1-973-972-3144, E-mail: pyrsopni@njms.rutgers.edu (NTP) or lingiava@njms.rutgers.edu (VAL) immunocompromised population. However, once organism sensitivities are discovered, narrowing of antibiotic regimens must occur to maintain good antibiotic stewardship.

Citation of this article: Lingiah VA, Pyrsopoulos NT. Bacterial infections in cirrhotic patients in a tertiary care hospital. J Clin Transl Hepatol 2021;000(000):000-000. doi: 10.14218/JCTH.2020.00076.

\section{Introduction}

Patients with cirrhosis are known to be immunocompromised and therefore more susceptible to infection. ${ }^{1}$ Among cirrhotic patients, $32-34 \%$ are diagnosed with bacterial infections on hospital admission or during their hospital course, significantly higher than the $5-7 \%$ overall infection rates for hospitalized patients in general. ${ }^{2}$ The incidence of infection rises to $45 \%$ in cirrhotic patients who are admitted for gastrointestinal hemorrhage. ${ }^{2}$ The mechanism behind the decrease in immune function has been reported to be multifactorial.

The liver plays a key role in immune defense as the largest organ of the reticuloendothelial system (RES). ${ }^{1}$ The liver comprises $90 \%$ of the RES, with its combined Kupffer and sinusoidal endothelial cell masses. In the setting of cirrhosis, the number of these specialized cells are decreased, leading to concurrent RES functional impairment and increased rates of bacteremia. ${ }^{1}$ This effect is compounded by the formation of portosystemic shunts which decrease blood flow through the liver and thereby avert the RES, allowing less bacteria and endotoxins to be removed from the circulation by the liver. ${ }^{1}$ It has also been shown that patients with cirrhosis have reduced neutrophil mobilization and phagocytic activity; this deficit correlates with the degree of liver disease. ${ }^{2}$ In addition, the aforementioned decreased phagocytic activity is compounded by decreased bactericidal and opsonization capacity. ${ }^{2}$ Moreover, cirrhotic patients have lower levels of protective proteins, including immunoglobulins (Igs)M, IgG, and IgA in ascitic fluid, as well as decreased concentrations of $\mathrm{C} 3, \mathrm{C} 4$, and $\mathrm{CH} 50$ in both the blood and ascitic fluid in combination, leading to further decreased bactericidal activity. ${ }^{2}$

In addition to an impaired immune system, cirrhotic patients are at higher risk for developing pathological bacterial translocation. In cirrhosis, the sympathetic nervous system is up-regulated, leading to slower gut motility and allowing bacterial stasis and overgrowth. Normally, there is a $100-1,000: 1$ ratio of anaerobes to Gram-negative bacilli (GNB) in the gut, with anaerobes rarely translocating across gut mucosa. But, due to the slower gut motility, the flora changes and GNB overpopulate. Portal hypertension 
causes intestinal submucosal edema, which disrupts the mucosal barrier, leading to increased permeability of these GNB across the intestinal walls into lymph nodes, the blood stream, and ultimately the initially sterile peritoneal fluid. ${ }^{1}$ Small intestinal bacterial overgrowth (SIBO) has also been associated with bacterial translocation and infection. ${ }^{3}$

The systemic inflammatory response syndrome (SIRS) is a process that cirrhotic patients are at higher risk for due to their elevated levels of endotoxins, proinflammatory cytokines (e.g., TNF-a and IL-6), as well as nitric oxide. SIRS contributes to immune dysregulation in cirrhotics, as well as other complications. Endotoxins like lipopolysaccharides from Gram-negative bacteria and peptidoglycans from Gram-positive bacteria also result in a considerable increase in pro-inflammatory cytokines that lead to significant inflammation. ${ }^{4}$ Cirrhotics have been shown to form higher levels of pro-inflammatory cytokines compared to non-cirrhotics when exposed to endotoxins. ${ }^{5}$ Increased nitric oxide production leads to oxidative stress and further vasodilatation. SIRS can lead to portal hypertension-related complications like variceal bleeding, hepatic encephalopathy, and renal failure. ${ }^{4}$ SIRS has been linked with sepsis in the past, when associated with infection, though the new Sepsis-3 criteria now focus on organ dysfunction due to abnormal host response to infection, as measured by the sequential organ failure assessment (commonly known as SOFA) and qSOFA scores. ${ }^{6,7}$

The gut microbiota has also been shown to be altered in cirrhosis. Studies have shown that the amount of beneficial autochthonous bacteria (e.g., Lachnospiraceae, Ruminococcaceae, and Clostridiales) are reduced in cirrhosis, with a subsequent increase in potentially pathogenic taxa (like Staphloccoccaeae, Enterobacteriaceae, and Enterococcaceae). A reduction in autochthonous taxa can be deleterious, as they produce short chain fatty acids that decrease colonic inflammation, contend with pathogenic bacteria for nutrients, create antibacterial peptides, and possibly improve the intestinal barrier. ${ }^{8}$

The most common bacterial infections in cirrhotic patients have been reported to be SBP $(25-31 \%)$, urinary tracts infections $(20-25 \%)$, pneumonia $(15-21 \%)$, bacteremia $(12 \%)$, and soft tissue infections $(11 \%) .{ }^{9}$ It has been reported that nearly $75 \%$ of bacterial infections in cirrhotic patients are caused by Gram-negative bacteria (like E. coli, Klebsiella, Pseudomonas, Vibrio, etc.), with Gram-positive bacteria comprising only $20.2 \%$ and anaerobes only $3.2 \% .{ }^{9}$ However, with increased use of quinolone prophylaxis, frequent hospitalizations, and the high number of invasive procedures cirrhotic patients undergo, there has been a shift to increasing numbers of infections caused by Gram-positive organisms (38-70\%). ${ }^{9}$ It has been reported that infections in cirrhotic patients encompass a 4-times higher risk of mortality compared to those in non-cirrhotics. ${ }^{10}$ In fact, cirrhotic patients who developed infections were shown to have a 1 -month mortality rate of $30 \%$ and a 1 -year mortality rate of $60 \% .{ }^{10}$

Given the high mortality rates associated with infections in cirrhotic patients and the changing types of infections, we decided to study the distribution of infections in one large academic center. The purpose of this retrospective chart review study was to assess the prevalence of infections in cirrhotic patients in a large urban academic liver center and investigate potential associations between infections, bacteria isolated, therapeutic regimens used, and mortality.

\section{Methods}

We performed a retrospective chart review of 263 patients who were admitted to the hepatology inpatient service at a tertiary care hospital. The protocol was reviewed and approved by the Rutgers New Jersey Medical School Institutional Review Board. Inclusion criteria were 1) adult patients between the ages of 18 and 80 years, and 2) diagnosis of cirrhosis. The diagnosis of cirrhosis was made radiographically or by histology. Patients were excluded from the study if they were 1) diagnosed with human immunodeficiency virus (commonly known as HIV) or acquired immune deficiency syndrome (commonly known as AIDS), 2) were on immunosuppressive medications after transplantation, or 3) had evidence of additional immunodeficiency syndromes or were on immunosuppressive agents for other disorders (i.e. patients with rheumatoid arthritis on steroids).

Infections were defined in the following ways. SBP was defined by a polymorphonuclear cell count in the ascitic fluid of $\geq 250$, with secondary bacterial peritonitis diagnosed by the same ascitic fluid findings after an abdominal procedure recently performed. Bacteremia was diagnosed by positive blood cultures and UTIs by urinalyses with 10 leukocytes or more and/or positive urine cultures. Respiratory tract infections were defined by chest $x$-rays with evidence of consolidation and/or positive sputum cultures, and cellulitis/other infections were noted by consistent physical exam findings. Diagnosis of other infections were made based on conventional criteria.

Blood and urine samples were collected as per University Hospital protocol. Blood cultures were collected peripherally in two sets of aerobic/anaerobic bottles from different sites, with cultures drawn from central lines only if there was high suspicion for line infections. Urine samples were drawn from patients via clean catch if the patient did not have a catheter placed, otherwise were drawn from urinary catheters if present. Straight catheterization was utilized if the patient was unable to void and did not have a catheter placed.

The electronic medical records were reviewed and data were extracted as follows: patient age, sex, etiology of cirrhosis, identification of bacteria isolated from cultures, multidrug-resistant (MDR) status, antibiotics administered, admission to intensive care unit (ICU), and patient mortality during or soon after hospitalization. Infections that were characterized as MDR included extended spectrum beta lactamase (ESBL) producing E. coli and Klebsiella species, methicillin-resistance Staphylococcus aureus (MRSA), E. faecium and $E$. faecalis resistant to vancomycin (VRE), and all bacteria resistant to at least three different antibiotics.

For statistical analysis, continuous variables were summarized as median (interquartile range) and categorical variables were summarized as percentages. Distributions of continuous variables were tested for normality by the Kolmogorov-Smirnov test, and, if skewed, were in-transformed. For continuous variables, the linear trend across infection variable (no infection, infection, MDR infection) was determined by analysis of variance; categorical variables were tested by the $x^{2}$ trend test. Binary logistic regression analysis was applied to assess the association between patients' clinical data and in-hospital mortality. Covariates that were univariably associated with in-hospital mortality (exploratory $p<0.10$ ) were entered into a multivariable model using the stepwise backward likelihood ratio method. All analyses were performed with a complete dataset using SPSS software (SPSS 27.0; IBM Corp., Armonk, NY, USA). All tests were two-tailed, and $p$ values $<0.05$ were considered statistically significant.

\section{Results}

A total of 263 patient charts were reviewed from patients admitted to the hepatology inpatient service from 2008- 


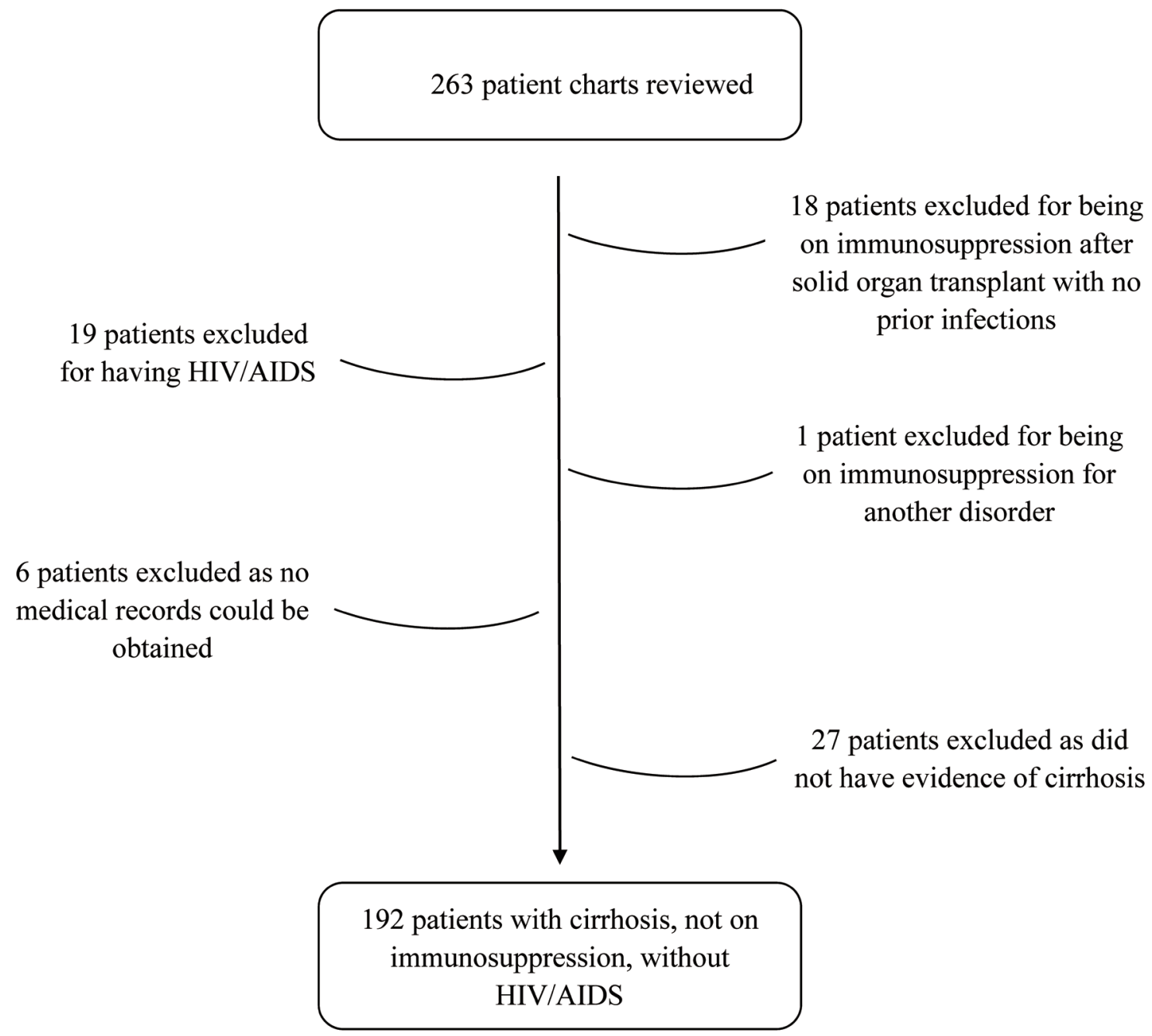

Fig. 1. Patient selection process.

2014. Eighteen patients were excluded for being on immunosuppressive medications after solid organ transplant, with no pre-transplant infections. An additional nineteen patients were excluded for carrying a diagnosis of HIV or AIDS. One patient was excluded for being on immunosuppressive medications for another disorder, and six patients were excluded as there was no information that could be found in the medical records for them. Twenty-seven patients could not be included as they did not have evidence of cirrhosis. This left 192 patients who met the criteria to be a part of the study (Fig. 1).

Men were more common than women, making up $62.6 \%$ of the study population. The mean age among patients diagnosed with infection was 55.8 years and 57.3 years among patients without infection. Hepatitis $C$ was the most common etiology of cirrhosis, both in patients with infection $(31.5 \%)$ and patients without infection $(29.7 \%)$, closely followed by alcohol (30.6\% in those with infections and $27.0 \%$ in those without infections). Other baseline data are mentioned in Table 1. The model for end-stage liver disease (MELD) scores were noted to be significantly higher in infected patients (whether MDR or non-MDR) compared to non-infected patients (22 and 20 vs. 13 respectively, $p<0.001)$. Ascites also was significantly more likely to be present in infected patients (whether MDR or non-MDR) compared to non-infected patients ( $89 \%$ and $73 \%$ vs. $53 \%$ respectively, $p<0.001$ ).

Infections were present in 105 (54.6\%) patients, and 60 $(31.2 \%)$ patients were noted to have multiple infections during a hospitalization or multiple hospitalizations for infections. A total of 201 infections were identified in these patients. UTIs were the most common infection identified $(37.8 \%)$, followed by bacteremia $(20.4 \%)$, pneumonia $(12.9 \%)$, SBP $(11.9 \%)$, abscess/cellulitis $(6.0 \%)$, infectious diarrhea (6.0\%), and other (5.0\%) (Fig. 2).

A total of 174 organisms were isolated from these infections, while 31 infections were culture-negative. E. coli was the most common bacteria isolated $(13.4 \%)$, both among sensitive and MDR infections (20.5\% in MDR infections and $11 \%$ in sensitive infections). Other prevalent bacteria isolated included Klebsiella (9\%), S. epidermidis $(6.5 \%)$, S. aureus (6\%), and VRE (6\%) (Fig. 3). Other frequent sensitive bacterial isolates included Klebsiella $(8.8 \%)$, S. aureus $(7.7 \%)$, and Clostridium difficile $(7.7 \%)$. Other prevalent MDR bacteria included VRE $(14.5 \%)$, Klebsiella $(12 \%)$, and S. epidermidis (10.8\%). MDR bacteria were the cause for $41.3 \%$ of all infections isolated. Fungi (Candida species and Aspergillus) were isolated in 19 cases, accounting for $9.5 \%$ of infections (Fig. 4). Fungemia, in particular, was present in five cases, or $2.5 \%$ of infections. Thirty-one infections 
Lingiah V.A. et al: Bacterial infections in cirrhotic patients

Table 1. Distribution of patients' clinical data and trends across different infection categories

\begin{tabular}{lllll}
\hline Clinical data & No infection & Infection & MDR infection & p-value* \\
\hline Age, median (IQR) & $58(50-63)$ & $56(53-63)$ & $56(51-61)$ & 0.49 \\
Male sex, \% & 69 & 58 & 62 & 0.36 \\
Diabetes mellitus, \% & 29 & 24 & 31 & 0.87 \\
MELD score, median (IQR) & $13(9-23)$ & $20(16-27)$ & $22(16-29)$ & $<0.001 *$ \\
Ascites, \% & 53 & 73 & 89 & $<0.001 *$ \\
Hepatic encephalopathy, \% & 32 & 49 & 50 & 0.051 \\
Rifaximin prior to admission, \% & 39 & 38 & 37 & 0.82 \\
\hline
\end{tabular}

${ }^{*} p$-value for trend; $p$-value $<0.05$ indicates statistical significance.

were noted to be culture-negative, with either pneumonia based on imaging findings $(n=10)$, SBP based on body fluid analysis $(n=7)$, cellulitis $(n=9)$, or UTI based on positive urinalysis $(n=5)$.

Vancomycin and piperacillin-tazobactam were the most commonly prescribed antibiotics (vancomycin in $40.8 \%$ of infections and Pip-Tazo in 39.8\%). Ceftriaxone and levofloxacin were used in $21.9 \%$ and $17.4 \%$ of infections respectively. Among the patients, $21.9 \%$ had decompensation from their infection(s) that required ICU care and $14.6 \%$ of patients died during hospitalization or soon after discharge. This is in comparison to the $29.7 \%$ of patients who had infections but did not require ICU stay or succumb to their infection. When reviewing factors that were associated with increased risk for mortality, MELD score, presence of ascites, and presence of infection (MDR or non-MDR) were found on univariate analysis to have a significant association. On multivariate analysis, MELD score [odds ratio (OR): 7.9, 95\% confidence interval (CI): 2.3-27.3, $p=0.001]$ and presence of infection (MDR or non-MDR) (MDR OR: 16.9, 95\% CI: $2.0-139.2, p=0.009$; non-MDR OR: 12.5 , 95\% CI: $1.5-105.6, p=0.02$ ) were found to be significantly associated with mortality (Table 2 ).

\section{Discussion}

Patients with cirrhosis are known to be at a higher risk of developing infections. This study showed that in our population of cirrhotic patients, slightly less than $55 \%$ had an infection on admission or developed one during hospitalization. This is a higher percentage than even prior papers suggest, emphasizing the delicate status of cirrhotic patients and that they are at high risk for developing infections and other forms of decompensation. These results may also have to do with our location as a tertiary care center in a large city. The fact that some of our patients had multiple hospitalizations with infections diagnosed may have also contributed to this higher percentage. These infections are made all the more critical given their significant association to patient death in this study. High priority should be given to prevention/early treatment of infections in cirrhotic patients, with appropriate, early broad antibiotic coverage in those suspected to have/with infections, given their associated worse outcomes.

Of interest was the large amount of fungal infections that were diagnosed. While often dismissed in immunocompetent

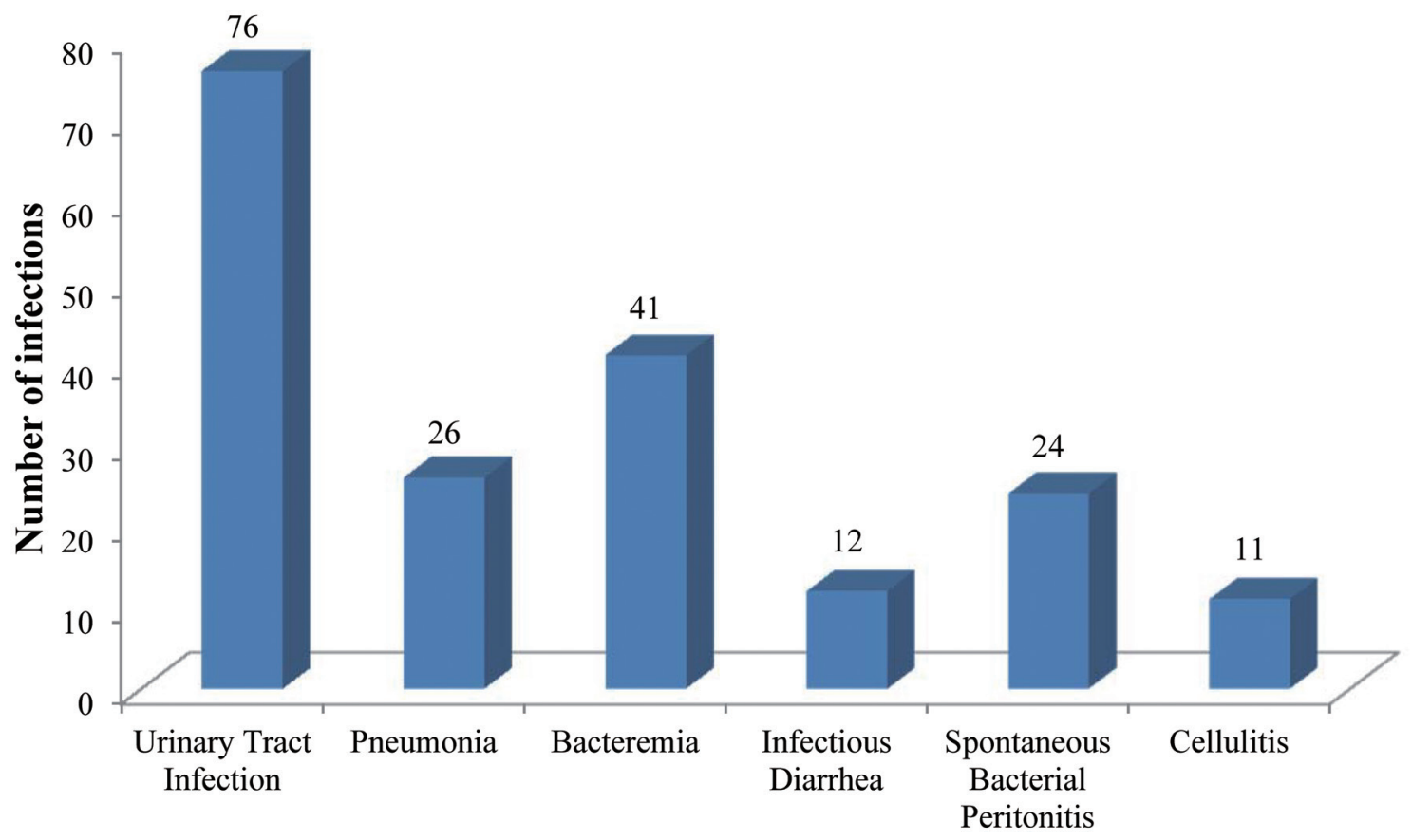

Fig. 2. Top six types of infections in cirrhotic patients. 
Lingiah V.A. et al: Bacterial infections in cirrhotic patients

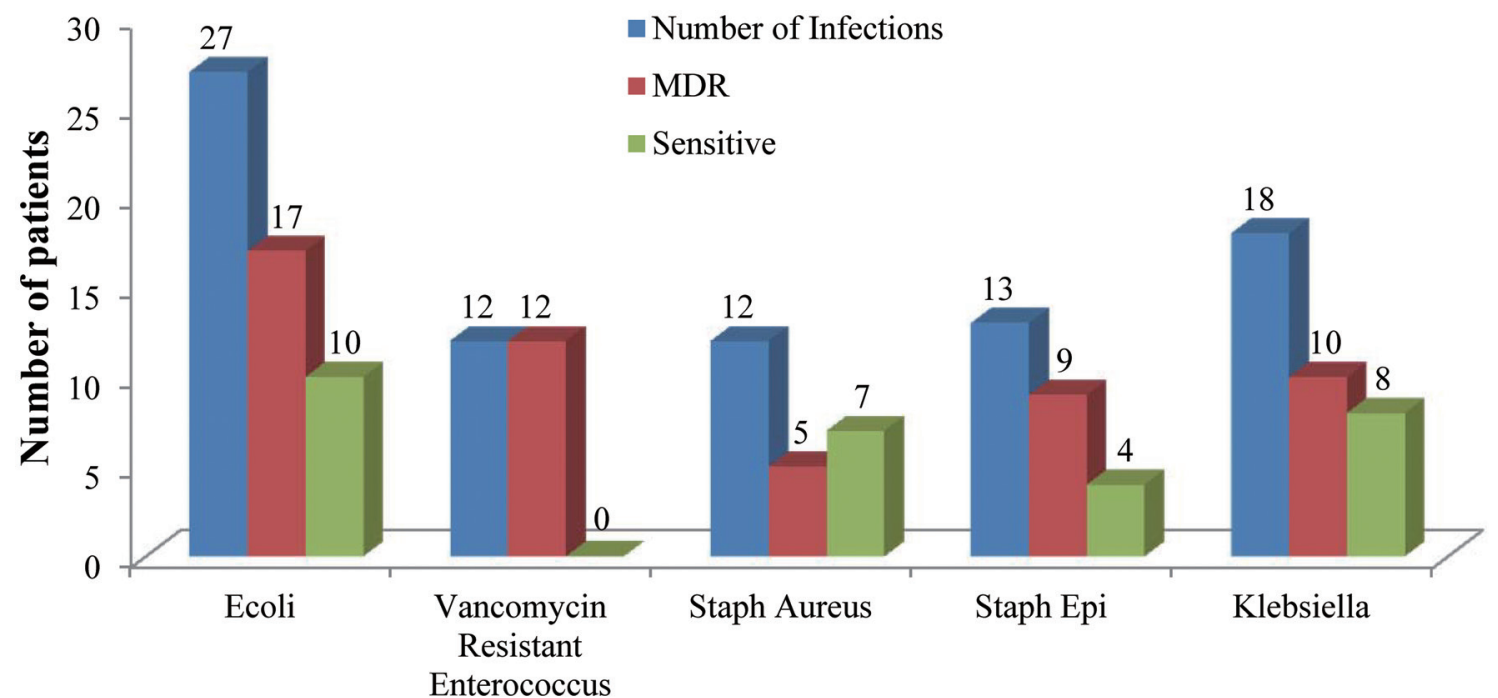

Fig. 3. Top five bacteria isolated from cirrhotic patients.

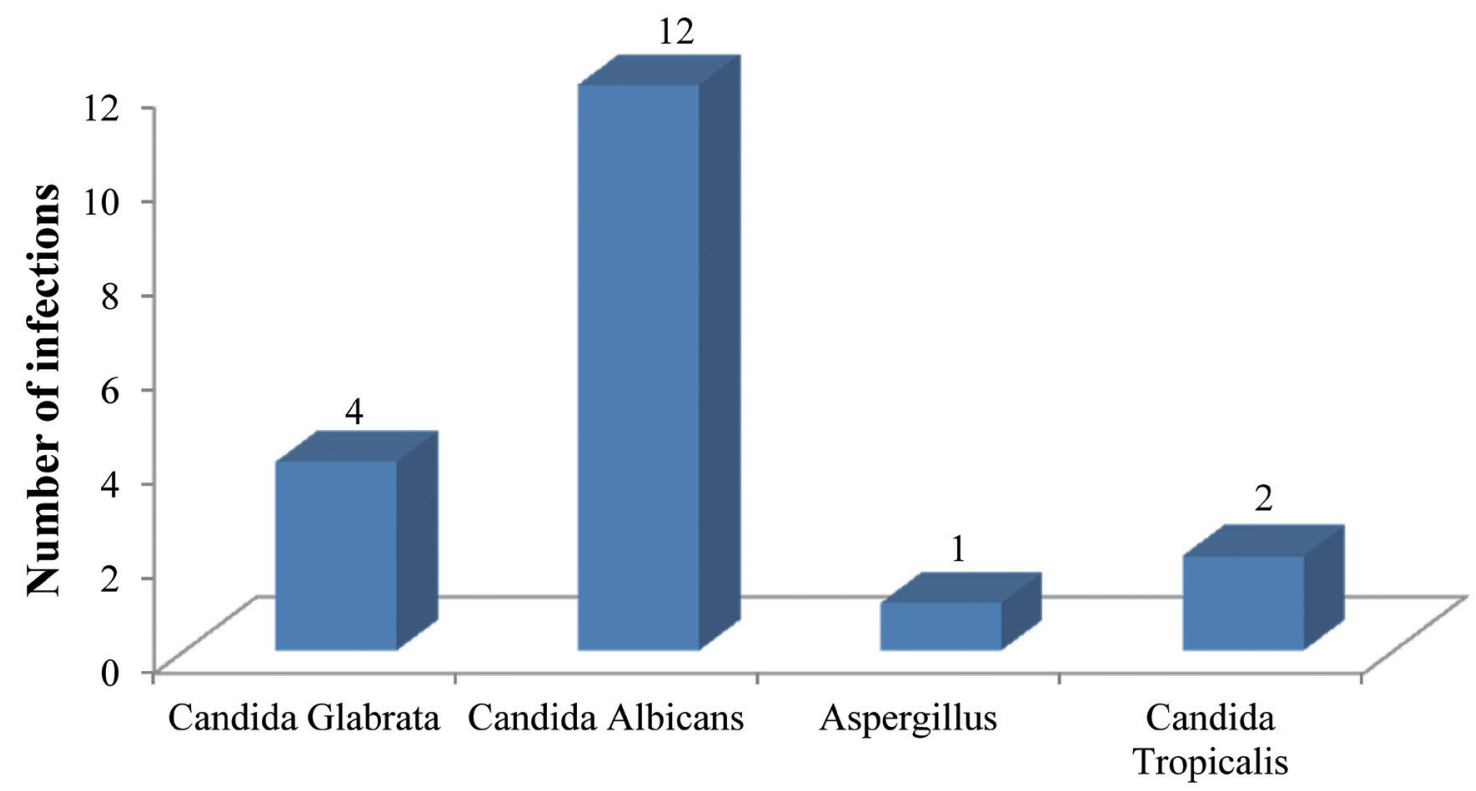

Fig. 4. Types of fungal infections in cirrhotic patients.

Table 2. Associations between patient's clinical data and in-hospital mortality

\begin{tabular}{|c|c|c|c|c|}
\hline & \multicolumn{2}{|c|}{ Univariable model } & \multicolumn{2}{|c|}{ Multivariable modela } \\
\hline & OR $(95 \% \mathrm{CI})$ & p-value* & OR (95\% CI) & p-value* \\
\hline MELD score ${ }^{b}$ & $11.3(3.7-34.2)$ & $<0.001 *$ & $7.9(2.3-27.3)$ & $0.001 *$ \\
\hline Ascites & $15.8(2.1-119.4)$ & $0.008 *$ & $7.3(0.9-61.1)$ & 0.07 \\
\hline Hepatic encephalopathy & $1.9(0.8-4.3)$ & 0.10 & $x$ & \\
\hline Rifaximin prior to admission & $2.2(1.0-4.8)$ & 0.06 & $x$ & \\
\hline Infection & $17(2.1-135.8)$ & $0.007 *$ & $12.5(1.5-105.6)$ & $0.020 *$ \\
\hline MDR infection & $29.7(3.8-232.3)$ & $0.001 *$ & $16.9(2.0-139.2)$ & $0.009 *$ \\
\hline
\end{tabular}

OR indicates odds ratio for dying during index hospitalization; $95 \% \mathrm{CI}$ indicates $95 \%$ confidence interval for the corresponding OR. aOnly clinical parameters with $p<0.10$ in univariate analysis are reported in the table. bPer 1 -Ln unit change in MELD score. ${ }^{*} p$-value $<0.05$ indicates statistical significance. $\mathrm{x}$ excluded in backward selection (i.e. not significant for prediction). 
patients as colonizers that are not responsible for sepsis/decompensation, we diagnosed numerous fungal infections, in particular many cases of fungemia, that were responsible for significant decompensation in our patients. These findings point to a gap in our management strategies for cirrhotic patients with suspected infections. While invasive fungal infections have been generally agreed to have serious complications in immunocompromised states, less focus has been given to infections regarded as fungal colonization. ${ }^{11,12}$ Lahmer et al., ${ }^{12}$ however, showed that even patients with fungal infections that did not meet criteria for invasive fungal infection (i.e. fungemia, biopsies of affected areas with fungal involvement, or chest x-ray/CT scan findings of lung or other organ) were still independently associated with higher mortality rates. Other studies have noted that the incidence of invasive fungal disease in the ICU was higher in patients with liver disease compared to other high-risk groups, though the mortality rates were not shown to differ. ${ }^{13}$

Although the above results make a compelling case for early and aggressive antibiotic use, it is clear that changes in the gut microbiome are occurring as a result of increasing antibiotic use. Bajaj et al. ${ }^{14}$ evaluated the difference in fungal and bacterial stool profiles of different populations. When comparing healthy controls to outpatient cirrhotic patients on/off antibiotics to hospitalized uninfected/infected culture-negative/infected culture-positive cirrhotic patients, there was a decrease in fungal diversity when comparing inpatients to outpatients, cirrhotics to healthy controls, and culture-positive infected inpatients to culture-negative infected patients or outpatients on antibiotics, with higher concentrations of Ascomycota species noted. As the more common type of fungal infections in cirrhosis (peritonitis, fungemia, and esophagitis) are often due to Ascomycota and portend a poor prognosis, antibiotics may exacerbate the incidence/risk of these infections.

A recent multicenter, prospective study from the North American Consortium for the Study of End-Stage Liver Disease (NACSELD) cohort evaluated inpatient cirrhotic patients to determine risk factors contributing the development of fungal infections and their significance on 30-day survival. Among hospitalized and infected patients, $12.7 \%$ were found to have fungal infections, all of which were nosocomial. Of the 134 fungal infections, 104 were noted after a prior bacterial infection and antibiotic use; however, the location of bacterial infection or type of bacteria isolated was not shown to significantly determine the development or location of fungal infections. This suggests antibiotic use for treatment of bacterial infections as a possible risk factor for the development of fungal infection. Other independent risk factors for fungal infections included diabetes, ICU admission, acute kidney injury, and bacterial infections diagnosed at admission. Fungal infections were also associated with worse 30-day survival. Most fungal infections had a $30 \%$ 30-day mortality rate, though fungemia and fungal peritonitis (forms of invasive fungal infections), had $>50 \%$ 30-day mortality rates. ${ }^{15}$

Close surveillance for fungal infections versus empiric antifungal coverage for patients with suspected infection and evidence of clinical deterioration or clinical deterioration despite antibiotic treatment of bacterial infections may help improve outcomes in this regard. While more is being learned at this time, further research into the topic of fungal infections in cirrhotic patients and surveillance, and potential prophylaxis is needed. ${ }^{16,17}$

Multidrug-resistant bacteria were isolated in over $41 \%$ of infections in our study. This correlates with the increasing rates of MDR infections being isolated in general and a change in the epidemiology of infections being isolated in cirrhotic patients. Studies have documented the increase in MDR infections, ranging from $23-47 \% .{ }^{18-20}$ Prior studies have shown that nosocomial infections, long-term nor- floxacin prophylaxis, infection by MDR bacteria in the last 6 months, and recent use of beta-lactam antibiotics have all been independently associated as risk factors for the development of MDR infections in cirrhotic patients. ${ }^{18}$ More recently, Piano et al. ${ }^{21}$ performed a multicenter, intercontinental, prospective study evaluating the prevalence of bacterial and fungal infections in patients with cirrhosis. Their study showed a global prevalence of MDR bacteria of $34 \%$. However, there were significant differences in MDR bacteria depending on geographic areas, with Asia having the highest prevalence. India had the highest prevalence, with $73 \%$ of bacteria isolated being MDR. This was attributed to over-the-counter access of antibiotics in the community and an increased presence of antibiotics in the environment. Independent risk factors for MDR bacterial infections were infections occurring in Asia (specifically India), antibiotic utilization 3 months prior to hospital admission, and site of infection. MDR infections were associated with decreased resolution rate of infection, higher incidence of shock and de novo organ failures, and higher in-hospital mortality compared to non-MDR infections. Interestingly, there was a lack of an association between quinolone prophylaxis and MDR infections. This may be due to the fact that only $10 \%$ patients in the study were on SBP prophylaxis, but bears further analysis. One of the most important findings was that giving appropriate empiric antibiotic coverage was independently associated with better in-hospital and 28-day survival rates. The efficacy of empiric antibiotics was the only modifiable predictor of mortality.

This increase in MDR infections has larger implications in regards to our antibiotic choices in this population of patients, namely, that patients' infections must be classified appropriately as community acquired, healthcare-associated, or nosocomial, so that the appropriate strength antibiotics can be started at admission. This is a critical time in the course of therapy, as empiric antibiotic regimens that are not effective in the initial $24-48$ hour period while cultures are being processed have been associated with increased mortality. ${ }^{22}$

In the past, third-generation cephalosporins had been the first choice of empiric treatment for SBP and several other infections, including UTIs. Studies have shown, however, evidence of bacterial resistance to third generation cephalosporins in $21.5 \%$ to $57 \%$ of SBP cases in Europe, with only $67 \%$ efficiency of empirical treatment with these agents. ${ }^{19}$ As a result, the European Association for the Study of the Liver (known as EASL) established guidelines advocating for stronger empiric antibiotics in nosocomial infections (piperacillin-tazobactam or meropenem +/glycopeptide), with mention that these choices should be tailored to the prevalent MDR bacteria of the local area. ${ }^{23}$ The most recent guidelines set by EASL recommend that healthcare-associated infections should be treated with carbapenems like nosocomial infections if the prevalence of MDR organisms is high in the area. They have also added piperacillin-tazobactam as a treatment option in community acquired infections. ${ }^{24}$ The efficacy of these guidelines has been shown in practice. Piano et al. ${ }^{25}$ looked at broaderspectrum antibiotic regimens in comparison to third generation cephalosporins in the treatment of nosocomial SBP, and found that meropenem with daptomycin was more effective than ceftazidime as empirical antibiotic treatment $(86.7 \%$ vs. $25 \%$ response to treatment, with $90 \%$ of ceftazidime non-responders responding to meropenem/daptomycin). The effectiveness of the initial empiric antibiotic treatment was noted to be a strong predictor of 90-day transplant-free survival in this patient population. The fact that $22 \%$ of the patients in our study required ICU care and nearly $15 \%$ succumbed to their illnesses is further testament to the need for broader empiric antibiotics for cirrhotic patients on presentation to the hospital with likelihood of sepsis. 
Moreover, new types of MDR infections are becoming more prevalent. Carbapenem-resistant Enterobacteriaceae (referred to as CRE) were uncommon prior to the year 2000 but have doubled in prevalence in the last decade among healthcare-associated infections. ${ }^{26}$ And, while healthcareassociated infections and nosocomial infections are where the majority of these cases have been noted in the past, studies are showing increasing rates of community acquired CRE infections, ranging from 7.7-29.5\% globally and from $5.6-10.8 \%$ in the USA. ${ }^{26,27}$ These findings bring to light the conundrum of antibiotic use in cirrhotic patients. Antibiotic prophylaxis and empiric broad spectrum antibiotic use has an important function, as it can prevent bacterial infections and positively impact liver decompensation and overall survival. However, it can lead to antibiotic resistance, potentially exacerbating the situation in the longer run. ${ }^{28}$ Understanding local antibiotic resistance patterns is important to tailoring empiric regimens that will improve survival outcomes without unnecessarily increasing risks for antibiotic resistance.

Given the increasing rates of MDR infections being isolated, with their associated mortality, it is important to consider new and innovative treatment and/or preventative options for bacterial infections in cirrhotic patients. Rifaximin has been evaluated in the recent past as a new option for prevention of SBP. Elfert et al. ${ }^{29}$ looked at 262 patients with cirrhosis and a prior episode of SBP, and randomly assigned them to receive either $1,200 \mathrm{mg}$ of rifaximin or $400 \mathrm{mg}$ of norfloxacin daily for 6 months. The rifaximin prophylaxis group was shown to have significantly lower rates of recurrent SBP when compared to the norfloxacin group $(3.9 \%$ vs. $14.1 \%, p=0.04)$. Additionally, there were 3 -times less encephalopathy-related deaths in the rifaximin group compared to the norfloxacin group. Menshawy et al. ${ }^{30}$ performed a meta-analysis of six studies including 973 patients to study the role of rifaximin in prevention of SBP. They found that rifaximin in addition to norfloxacin resulted in statistically lower rates of SBP [relative risk (RR): $0.58,95 \% \mathrm{CI}$ : $0.37-0.92, p=0.02]$ and hepatic encephalopathy (RR: 0.38 , 95\% CI: $0.17-0.84, p=0.02$ ), compared to the norfloxacin monotherapy group. They also found no significant differences between rifaximin and norfloxacin in regards to frequency of SBP (RR: $0.49,95 \%$ CI: $0.24-1.01, p=0.05$ ). Our own study did not see much of an impact of rifaximin on infection rates, with $37.4 \%$ of those with infections on rifaximin compared to $38.5 \%$ of those without infections.

This study has some limitations. It is a retrospective study, and so is at risk of selection and researcher bias. UTIs were noted to be the most common infection isolated, though a subset of these cases may have been related to asymptomatic bacteriuria, rather than actual infection. Our data also revealed a number of cases of $S$. epidermidis infections. While appropriate techniques were utilized in obtaining cultures, it is possible that contamination may have contributed to the number of these cases. Additionally, our patients included those of geriatric age group, whose immunosenescence may have put them at higher risk for the development of infections. It is also a single-center study in an urban tertiary care hospital, receiving referrals from neighboring hospitals of decompensated cirrhotic patients, and may not be fully generalizable to different demographic centers. Further prospective, multicenter trials can help to corroborate these findings in a more substantial way.

In short, our study shows that the incidence of infections in cirrhotic patients is much higher than that of their non-cirrhotic counterparts, and even higher than prior studies of cirrhotic patients suggest. As a large component of these infections are caused by MDR bacteria and fungal organisms, stronger empiric antibiotics and antifungals need to be considered when initially treating this immunocompromised population who may not mount appropriate im- munologic responses to these pathogens. However, once organism sensitivities have been discovered, narrowing of antibiotic regimens needs to occur to maintain good antibiotic stewardship.

\section{Funding}

None to declare.

\section{Conflict of interest}

Pyrsopoulos NT is a recipient of research grants from Mallinckrodt, Valeant, Gilead, Bayer, Beigene, Genfit, Prometheus, Grifols, Intercept, Zydus and Eisai, which are outside the submitted work. Lingiah VA has no conflict of interests related to this publication.

\section{Author contributions}

Reviewed the literature, performed the majority of data acquisition and analysis, manuscript preparation, and preparation of figures and tables (VAL), designed, co-authored and revised the manuscript (NTP).

\section{References}

[1] Ghassemi S, Garcia-Tsao G. Prevention and treatment of infections in patients with cirrhosis. Best Pract Res Clin Gastroenterol 2007;21:77-93. doi:10.1016/j.bpg.2006.07.004.

[2] Bonnel AR, Bunchorntavakul C, Reddy KR. Immune dysfunction and infections in patients with cirrhosis. Clin Gastroenterol Hepatol 2011;9:727738. doi:10.1016/j.cgh.2011.02.031.

[3] Wiest R, Lawson M, Geuking M. Pathological bacterial translocation in liver cirrhosis. J Hepatol 2014;60:197-209. doi:10.1016/j.jhep.2013.07.044.

[4] Noor MT, Manoria P. Immune dysfunction in cirrhosis. J Clin Transl Hepatol 2017;5:50-58. doi:10.14218/JCTH.2016.00056.

[5] Byl B, Roucloux I, Crusiaux A, Dupont E, Devière J. Tumor necrosis factor alpha and interleukin 6 plasma levels in infected cirrhotic patients. Gastroenterology 1993:104:1492-1497. doi:10.1016/0016-5085(93)90361-f.

[6] Singer M, Deutschman CS, Seymour CW, Shankar-Hari M, Annane D, Bauer $M$, et al. The third international consensus definitions for sepsis and septic shock (sepsis-3). JAMA 2016;315:801-810. doi:10.1001/ jama.2016.0287.

[7] Simpson SQ. SIRS in the time of sepsis-3. Chest 2018;153:34-38. doi:10.1016/j.chest.2017.10.006.

[8] Bajaj JS, Heuman DM, Hylemon PB, Sanyal AJ, White MB, Monteith P, et al. Altered profile of human gut microbiome is associated with cirrhosis and its complications. J Hepatol 2014;60:940-947. doi:10.1016/j. jhep.2013.12.019.

[9] Bunchorntavakul C, Chavalitdhamrong D. Bacterial infections other than spontaneous bacterial peritonitis in cirrhosis. World J Hepatol 2012;4:158168. doi:10.4254/wjh.v4.i5.158.

[10] Arvaniti V, D'Amico G, Fede G, Manousou P, Tsochatzis E, Pleguezuelo $\mathrm{M}$, et al. Infections in patients with cirrhosis increase mortality fourfold and should be used in determining prognosis. Gastroenterology 2010;139:1246-1256. 1256.e1-5doi:10.1053/j.gastro.2010.06.019.

[11] Hassan EA, Abd El-Rehim AS, Hassany SM, Ahmed AO, Elsherbiny NM, Mohammed $\mathrm{MH}$. Fungal infection in patients with end-stage liver disease: low frequency or low index of suspicion. Int J Infect Dis 2014;23:69-74. doi: $10.1016 / j$.ijid.2013.12.014.

[12] Lahmer T, Messer M, Mayr U, Saugel B, Noe S, Schultheiss C, et al. Fungal "colonisation" is associated with increased mortality in medical intensive care unit patients with liver cirrhosis. Mycopathologia 2015;179:63-71. doi:10.1007/s11046-014-9825-6

[13] Theocharidou E, Agarwal B, Jeffrey G, Jalan R, Harrison D, Burroughs AK, et al. Early invasive fungal infections and colonization in patients with cirrhosis admitted to the intensive care unit. Clin Microbiol Infect 2016:22:189. e1-189.e7. doi:10.1016/j.cmi.2015.10.020

[14] Bajaj JS, Liu EJ, Kheradman R, Fagan A, Heuman DM, White M, et al. Fungal dysbiosis in cirrhosis. Gut 2018;67:1146-1154. doi:10.1136/ gutjnl-2016-313170.

[15] Bajaj JS, Reddy RK, Tandon P, Wong F, Kamath PS, Biggins SW, et al. Prediction of fungal infection development and their impact on survival using the NACSELD cohort. Am J Gastroenterol 2018;113:556-563. doi:10.1038/ajg.2017.471.

[16] Majeed A, Ullah W, Zahid U, Al Mohajer M. Persistent spontaneous fungal peritonitis secondary to Candida albicans in a patient with alcoholic cirrho- 
Sis and review of the literature. BMJ Case Rep 2016;2016:bcr2016216979. doi:10.1136/bcr-2016-216979.

[17] Shizuma T. Spontaneous bacterial and fungal peritonitis in patients with liver cirrhosis: A literature review. World J Hepatol 2018;10:254-266. doi:10.4254/wjh.v10.i2.254.

[18] Fernández J, Acevedo J, Castro M, Garcia O, de Lope CR, Roca D, et al. Prevalence and risk factors of infections by multiresistant bacteria in cirrhosis: a prospective study. Hepatology 2012;55:1551-1561. doi:10.1002/ Sis: a prospect
hep. 25532 .

[19] Costabeber AM, Mattos AA, Sukiennik TC. Prevalence of bacterial resistance in hospitalized cirrhotic patients in southern Brazil: A new challenge. Rev Inst Med Trop Sao Paulo 2016;58:36. doi:10.1590/S1678-9946201658036.

[20] Tandon P, Delisle A, Topal JE, Garcia-Tsao G. High prevalence of antibioticresistant bacterial infections among patients with cirrhosis at a US liver center. Clin Gastroenterol Hepatol 2012;10:1291-1298. doi:10.1016/j. cgh.2012.08.017.

[21] Piano S, Singh V, Caraceni $P$, Maiwall R, Alessandria C, Fernandez J, et al. Epidemiology and effects of bacterial infections in patients with cirrhosis worldwide. Gastroenterology 2019;156:1368-1380.e10. doi:10.1053/j. worldwide. Gastroenter
gastro.2018.12.005

[22] EASL clinical practice guidelines on the management of ascites, spontaneous bacterial peritonitis, and hepatorenal syndrome in cirrhosis. J Hepatol 2010;53:397-417. doi:10.1016/j.jhep.2010.05.004.

[23] Jalan R, Fernandez J, Wiest R, Schnabl B, Moreau R, Angeli P, et al. Bacterial infections in cirrhosis: a position statement based on the EASL Special Conference 2013. J Hepatol 2014;60:1310-1324. doi:10.1016/j. jhep.2014.01.024.

[24] EASL Clinical Practice Guidelines for the management of patients with decompensated cirrhosis. J Hepatol 2018;69:406-460. doi:10.1016/j. jhep.2018.03.024.

[25] Piano S, Fasolato S, Salinas F, Romano A, Tonon M, Morando F, et al. The empirical antibiotic treatment of nosocomial spontaneous bacterial peritonitis: Results of a randomized, controlled clinical trial. Hepatology 2016;63:1299-1309. doi:10.1002/hep.27941.

[26] Kelly AM, Mathema B, Larson EL. Carbapenem-resistant Enterobacteriaceae in the community: a scoping review. Int $]$ Antimicrob Agents 2017;50:127-134. doi:10.1016/j.ijantimicag.2017.03.012

[27] Alexopoulou A, Papadopoulos N, Eliopoulos DG, Alexaki A, Tsiriga A, Toutouza $M$, et al. Increasing frequency of gram-positive cocci and gram-negative multidrug-resistant bacteria in spontaneous bacterial peritonitis. Liver Int 2013;33:975-981. doi:10.1111/liv.12152.

[28] Zuccaro V, Columpsi P, Sacchi P, Lucà MG, Fagiuoli S, Bruno R. Antibiotic stewardship and empirical antibiotic treatment: How can they get along? Dig Liver Dis 2017;49:579-584. doi:10.1016/j.dld.2017.01.157.

[29] Elfert A, Abo Ali L, Soliman S, Ibrahim S, Abd-Elsalam S. Randomizedcontrolled trial of rifaximin versus norfloxacin for secondary prophylaxis of spontaneous bacterial peritonitis. Eur J Gastroenterol Hepatol 2016;28:1450-1454. doi:10.1097/MEG.0000000000000724.

[30] Menshawy A, Mattar O, Barssoum K, AboEI-Naga AM, Salim HM, Mohamed AMF, et al. Safety and Efficacy of Rifaximin in Prophylaxis of Spontaneous Bacterial Peritonitis: A Systematic Review and Meta-analysis. Curr Drug Targets 2019;20:380-387. doi:10.2174/1389450119666180924145156. 